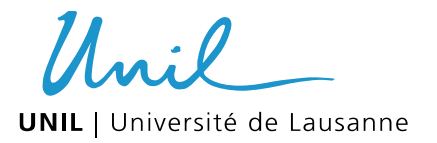

Faculté de biologie

et de médecine
Centre

Universitaire

Romand

de Médecine Légale

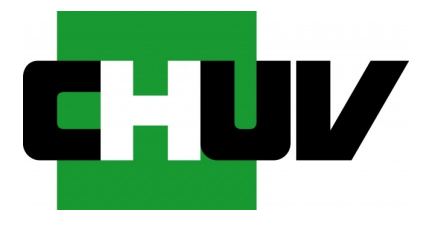

Mémoire de Maîtrise en médecine No 4335

\title{
Usefulness of liver function tests in postmortem samples
}

\author{
Etudiant \\ Fumeaux Léonard
}

Tuteur

Dr. Palmiere Cristian

Centre Universitaire Romand de Médecine Légale

\author{
Expert \\ Prof. Waeber Gérard \\ Service de médecine interne CHUV
}

Lausanne, le 15 décembre 2017 
Usefulness of liver function tests in postmortem samples

Abstract

Liver function tests have been investigated in the forensic setting in several biological fluids collected at autopsy. Nevertheless, the results of these investigations have provided diverging information on postmortem stability of liver function markers and postmortem reliability of liver function assessment. The first aim of this study was to determine gamma-glutamyltransferase, alanine aminotransferase, aspartate aminotransferase, alkaline phosphatase, total bilirubin, total proteins, and albumin in antemortem and postmortem serum samples in a series of cases that underwent forensic investigations and had both samples available. The second aim was to measure the same molecules in cerebrospinal fluid. Preliminary results indicated that femoral (and cardiac) blood postmortem serum concentrations of bilirubin, total proteins, and albumin, as well as femoral blood postmortem serum concentrations of gamma-glutamyltransferase, can be considered to reliably reflect antemortem serum concentrations, thus suggesting that postmortem values could be used as surrogates for antemortem levels. Conversely, alanine aminotransferase, alkaline phosphatase and bilirubin levels in cerebrospinal fluid stored in both preservative-free and sodium fluoride tubes are of no value to estimate their antemortem serum concentrations, irrespective of liver disease severity found at autopsy.

Keywords: Postmortem biochemistry; Autopsy; Liver function tests; Postmortem serum; Cerebrospinal fluid. 
Introduction

Liver disease is a major cause of illness and death worldwide. Vaccination campaigns against hepatitis B virus (HBV) and mandatory hepatitis $\mathrm{C}$ virus (HCV) screening for blood transfusion have resulted in a substantial decline in the number of newly HBV- and HCV-infected patients in several countries. At the same time, the number of patients with alcohol-related liver disease has increased because of changes in people's lifestyles and heavier alcohol consumption. Moreover, late-stage liver diseases, such as cirrhosis, chronic liver failure and hepatocellular carcinoma, have increased two- to threefold over the past 20 years, leading to high mortality from chronic liver diseases. ${ }^{1-3}$

The major causes of liver disease include excess chronic alcohol consumption, virus infections, autoimmune disorders, therapeutic drugs, and non-alcoholic accumulation of fat deposits in hepatocytes. ${ }^{1,3}$

In the clinical setting, hepatic function assessment in patients with liver disease is based on laboratory investigations of serum biochemical parameters prior to confirmation by histopathology. Conversely, evaluation of liver function based on postmortem laboratory investigations is mainly limited to advanced liver disease, specific biological samples collected at autopsy and selected laboratory biomarkers, some of those used in the living being considered of no value after death due to postmortem autolysis and decompositional change onset. ${ }^{4,5}$

Gamma-glutamyltransferase (GGT), alanine aminotransferase (ALT), aspartate aminotransferase (AST), alkaline phosphatase (ALP) and bilirubin, along with total proteins and albumin, have been occasionally investigated in the past in antemortem and postmortem serum (from peripheral and cardiac blood) samples, vitreous humor, urine and cerebrospinal fluid in both humans and animals by some research teams. The results of these studies, though interesting, have provided sometimes diverging conclusions on postmortem molecule stability and postmortem measurement reliability, leading to the conclusion 
that hepatic function assessment from postmortem laboratory investigations is of limited value or even none whatsoever. ${ }^{5-13}$

The purpose of the study presented herein was twofold: a) to determine GGT, ALT, AST, ALP, total bilirubin, total proteins, and albumin in a series of cases that underwent postmortem investigations with antemortem and postmortem serum samples available for comparison, thereby investigating the postmortem stability of these compounds in blood after death, b) measure the same molecules in cerebrospinal fluid in a series of cases that underwent medicolegal investigations, thereby evaluating the distribution of these molecules in this biological sample as well as the usefulness of their determination in this specimen for diagnostic purposes in the forensic setting.

Materials and methods

Study design

The present study was performed in 2016-2017, designed as a single centre study. All cases selected for the study originated from forensic practice and underwent medicolegal autopsies as requested by local inquiring authorities (the public prosecutor). Laboratory analyses were performed as part of the medicolegal investigations.

Study populations

To address the first purpose of the study, comparisons between antemortem and postmortem serum levels of GGT, ALT, AST, ALP, total bilirubin, total proteins, and albumin were performed on samples 
collected from 10 individuals (8 males and 2 females, with a mean age of 48 years, range 39-69 years) who had been admitted to hospital shortly prior to death.

Diagnosis on admission included sudden loss of consciousness in the presence of witnesses. Cardiopulmonary resuscitation attempts were performed on-site by co-workers, bystanders, and laypersons until paramedics arrived. Information pertaining to preexisting liver disease and other conditions potentially affecting blood levels of these molecules was unavailable at hospital admission. Antemortem samples consisted of serum samples obtained rapidly on admission to hospital. Death was pronounced in all cases approximately within one hour of arrival.

Medicolegal autopsies were performed between 12 and $48 \mathrm{~h}$ after death. Postmortem samples consisted of postmortem serum samples obtained from peripheral (femoral blood) prior to autopsy and from cardiac blood (right cardiac atrium) during autopsy.

Causes of death at postmortem investigations included cardiac deaths ( 7 cases) and drug intoxication ( 3 cases).

To address the second aim of the study, comparisons between postmortem serum (from femoral blood) samples and cerebrospinal fluid levels of GGT, ALT, AST, ALP, and total bilirubin were performed on samples collected from 15 individuals ( 9 males and 6 females, with a mean age of 56 years, range 26-68 years), whose deaths occurred outside the hospital. Complete medicolegal autopsies were performed between 18 and $48 \mathrm{~h}$ after death.

All cases were characterized by the presence of hepatic steatosis and cirrhosis, histologically confirmed. Abdominal ascites and jaundice were observed in 6 out of 15 cases, bleeding esophageal varices in 2 out of 15 cases, hepatocellular carcinoma (histologically confirmed) in 1 out of 15 cases.

Causes of death included natural deaths (cardiac death or death following complications of end-stage liver disease, 9 out of 15 cases) and drug intoxication (6 out of 15 cases). 
Case inclusion criteria for the first study group consisted of antemortem and postmortem serum availability (from both femoral and cardiac blood) as well as absence of intravenous fluid administration or minimal intravenous fluid administration.

Case inclusion criteria for the second study group consisted of postmortem serum (from femoral blood) and cerebrospinal fluid availability at autopsy.

Postmortem investigations and sample collection

Medicolegal autopsies, histology, toxicology, and biochemical investigations were performed in all cases. Conventional histology included haematoxylin-eosin (HE) stains of brain, heart, lung, liver, and kidney samples. HE stain was performed after tissue fixation in formaldehyde.

Systematic toxicological analysis included blood ethanol level determination as well as general screening for volatile and nonvolatile drugs, poisons, and metabolites.

Peripheral blood from the femoral veins was systematically collected for toxicological and biochemical studies prior to autopsy. Femoral blood samples were collected by aspiration with sterile needles and syringes from the femoral vein(s). Blood samples were drawn after clamping the vein(s) at the proximal end and keeping the lower limb(s) raised for several minutes. Samples were stored in tubes containing sodium fluoride and preservative-free gel serum separator tubes. The latter were centrifuged immediately post collection at $3000 \mathrm{~g}$ for $15 \mathrm{~min}$. After centrifugation, the separated supernatant (postmortem serum) was collected and stored in preservative-free tubes. No specimens were excluded due to insufficient sample volume. Postmortem serum samples were transferred to the laboratories immediately post collection. When analyses were delayed, samples were stored at $-20^{\circ} \mathrm{C}$.

Blood from the right cardiac atrium was drawn using sterile syringes after opening the pericardium at autopsy. Blood samples were collected and stored in tubes containing sodium fluoride and preservative- 
free gel serum separator tubes. The latter were centrifuged immediately post collection at $3000 \mathrm{~g}$ for 15 min. After centrifugation, the separated supernatant (postmortem serum) was collected and stored in preservative-free tubes. No specimens were excluded due to insufficient sample volume. Postmortem serum samples were transferred to the laboratories immediately post collection. When analyses were delayed, samples were stored at $-20^{\circ} \mathrm{C}$.

Undiluted cerebrospinal fluid samples were collected by aspiration using a sterile needle and syringe by suboccipital puncture as soon as possible upon arrival of the bodies at the morgue and prior to autopsy. All samples were immediately centrifuged at $3000 \mathrm{~g}$ for $15 \mathrm{~min}$. After centrifugation, the separated supernatant was collected and stored in tubes containing sodium fluoride and preservative-free tubes. Cerebrospinal fluid samples were transferred to the laboratories immediately post collection. When analyses were delayed, samples were stored at $-20^{\circ} \mathrm{C}$. No specimens were excluded due to insufficient sample volume.

Laboratory assays

Albumin concentration was determined by the bromocresol green method. Total proteins were determined by the biuret method. GGT, ALP, AST and ALT were determined by standard procedures (International Federation of Clinical Chemistry and Laboratory Medicine (IFCC) and Swiss Society of Clinical Chemistry (SSCC) reference measurement procedure at $37^{\circ} \mathrm{C}$ ). Total bilirubin was determined by DPD dichloro-2,5-benzene diazonium method. 
Statistical analysis

Concerning the first purpose of the study, comparisons of GGT, ALT, AST, ALP, total bilirubin, total protein, and albumin levels in antemortem and postmortem serum samples (from peripheral and cardiac blood) were performed using the Student's t test.

Concerning the second aim of the study, comparison of GGT, ALT, AST, ALP, and total bilirubin levels in postmortem serum (from femoral blood) and cerebrospinal fluid samples were performed using the Student's t test.

Statistical significance was defined as a $P$ value of less than 0.05 . All statistical analyses were performed using GraphPad Prism 4.0 (GraphPad Software, La Jolla, CA, USA).

Results

Concerning the first aim of this study, no statistically significant differences were noticed between antemortem and postmortem serum (from both femoral and cardiac blood) levels of total proteins, albumin, and bilirubin, whereas statistically significant differences were observed between antemortem and postmortem serum (from both femoral and cardiac blood) levels of ALT and AST $(P<0.05$ for both biomarkers in both postmortem serum samples versus antemortem serum).

Postmortem serum ALT and AST levels from both postmortem blood samples were systematically higher than antemortem serum levels, irrespective of the postmortem interval.

Concerning ALP and GGT specifically, measured levels in both postmortem serum samples were systematically higher than those measured in antemortem serum, irrespective of the postmortem interval. Moreover, postmortem serum from right cardiac atrium blood values of both biomarkers were systematically (and erratically) higher than the corresponding postmortem serum from femoral blood 
values, irrespective of the postmortem interval, with no relationship between postmortem serum from cardiac blood values versus postmortem serum from femoral blood values. Statistically speaking, we observed a significant increase in ALP values in both postmortem serum samples $(P<0.05$ for both postmortem serum samples versus antemortem serum) and GGT values $(P<0.05)$ in postmortem serum from cardiac blood versus antemortem serum, whereas GGT values in postmortem serum from femoral blood did not differ significantly from those in antemortem serum.

Concerning the second aim of the study (comparisons between postmortem serum from femoral blood and cerebrospinal fluid levels of total bilirubin, ALT, AST, ALP, and GGT), measurable bilirubin levels as well as ALP and ALT activities were demonstrable in cerebrospinal fluid samples in all studied cases $(\mathrm{N}=$ 15). Nevertheless, cerebrospinal fluid to postmortem serum ratios varied widely and unpredictably. No statistically significant differences were observed between measured levels of bilirubin, ALP and ALT in cerebrospinal fluid samples stored in preservative-free tubes versus samples stored in tubes containing sodium fluoride.

\section{Discussion}

One of the first studies pertaining to liver function assessment based on postmortem laboratory investigations (bilirubin, proteins, albumin, and ALP) was performed by Naumann ${ }^{10}$, who used postmortem serum obtained from blood collected from the inferior vena cava in 131 cases. What is interesting in this study is that "normal postmortem values" of these markers were established based on laboratory investigations performed in individuals without liver disease or conditions known to affect the results of these tests. In such individuals, postmortem serum total, direct, and indirect bilirubin as well as total protein and albumin values corresponded closely with the values usually regarded as normal in the living. Postmortem ALP values varied over a wide range, though the average was only 
slightly higher than the normal upper limit during life. On the other hand, postmortem serum levels of both proteins and ALP did not correlate closely enough with liver disease to be of value in individual cases.

In a subsequent study, Naumann and Young ${ }^{14}$ found that total and direct bilirubin were demonstrable in cerebrospinal fluid, albeit in lesser amounts than in serum (cerebrospinal fluid to serum ratio of 1:35 and $1: 45$, respectively).

Succeeding Naumann ${ }^{10}$, Enticknap ${ }^{15}$ and Coe ${ }^{16}$ investigated ALP levels in postmortem serum and found significantly increases of up to ten times normal antemortem values $48 \mathrm{~h}$ after death.

ALT concentrations were shown to progressively increase after death in peripheral blood, with a rise that was roughly linear with the postmortem interval in the first 60 hours after death. In addition, detectable ALT levels were noticed in cerebrospinal fluid, with concentrations progressively increasing after death. However, accurate assessments of postmortem interval based on ALT measurement in cerebrospinal fluid were considered unfeasible due to the wide range of obtained values. ${ }^{15,17,18}$

In a case series including 94 individuals with normal antemortem serum bilirubin levels, Coe ${ }^{5,6,16}$ observed a small though definite augmentation in average serum bilirubin concentration with increasing postmortem intervals. On the other hand, increases in serum bilirubin concentrations in icteric individuals were relatively small, enabling one to determine the degree on antemortem jaundice from postmortem values.

In a study involving animals, Schoning and Strafuss ${ }^{8}$ determined antemortem and postmortem serum levels of ALP, ALT and GGT as well as cerebrospinal fluid ALP and ALT levels. They observed that ALT and GGT levels significantly increased in serum after death. Postmortem serum concentrations of these markers varied void of correlation with postmortem interval or ambient temperature, as did ALP and ALT values in cerebrospinal fluid. Conversely, postmortem serum ALP levels increased as early as $3 \mathrm{~h}$ 
after death and rose continually with postmortem intervals. Temperature also affected ALP values, with levels that were higher at $37^{\circ} \mathrm{C}$ than $4^{\circ} \mathrm{C}$.

Lythgoe ${ }^{12,19}$ determined ALP (and lactate dehydrogenase) in postmortem serum obtained from blood samples collected at different sampling sites (upper and lower limbs as well as both sides of the heart) and concluded that ALP activity in the peripheral regions was slightly elevated after death (with values independent of the time of death), possibly as a result of ALP release from leucocytes. In postmortem serum samples obtained from the right heart blood, ALP values were high and unpredictable, possibly owing to postmortem blood movement that could carry the enzymes released from the liver to the right heart cavities.

Devgun and Dunbar ${ }^{9,20}$ measured a series of biomarkers, including hepatic biomarkers, in vitreous humor and observed that only very low levels of ALT, GGT, ALP, total proteins, albumin and bilirubin could be find in this sample, whereas AST determination showed appreciable vitreous levels, possibly related to sudden release of hyalocyte contents at death. Piette and De Schrijver ${ }^{21}$ obtained similar results concerning GGT activity in vitreous.

The usefulness of GGT determination in postmortem serum has been investigated by Piette and De Schrijver ${ }^{21}$, Uemura et al. ${ }^{7}$, and Michiue et al. ${ }^{11}$. Piette and De Schrijver ${ }^{21}$ found that GGT enzymatic activity in postmortem serum (obtained from subclavian vein or femoral vein blood) remained remarkably stable until $72 \mathrm{~h}$ after death. GGT activity was more pronounced at right heart level, similar to what was previously described by Lythgoe ${ }^{12,19}$ for ALP and lactate dehydrogenase. Moreover, enzyme activity at femoral vein level was less elevated than that of the left heart or subclavian vein levels. Based on their results, these authors concluded that GGT determination after death proved useful to investigate chronic alcohol abuse in the postmortem setting. Similar results were obtained by Uemura et al. ${ }^{7}$, who measured GGT levels in postmortem serum samples obtained from blood collected at different sampling sites (right and left heart cavities as well as femoral vein within $72 \mathrm{~h}$ postmortem). These 
authors observed that postmortem increases of GGT levels were less pronounced at the femoral vein level, which was therefore recommended as preferential blood sampling site for GGT activity determination after death. Michiue et al. ${ }^{11}$ measured GGT activity in 163 cases (within about $62 \mathrm{~h}$ postmortem and no preexisting liver or pancreatic disease or other conditions potentially affecting GGT levels) in postmortem serum obtained from left and right heart chambers and observed levels below $300 \mathrm{U} / \mathrm{L}$ in most cases. These results were irrespective of the postmortem interval, though right cardiac postmortem serum values were higher than left cardiac postmortem serum values in some cases.

Results obtained from Arroyo et al. ${ }^{13}$ pertaining to human cerebrospinal fluid $(50 \mathrm{~h}$ postmortem interval) documented ALP activity in this fluid, though with statistically significant differences among the studied groups that were difficult to interpret.

The results of the study presented herein appear to corroborate those reported in former investigations in the realm of forensic pathology on this topic and indicate that that femoral (and cardiac) blood postmortem serum concentrations of bilirubin, total proteins, and albumin, as well as femoral blood postmortem serum concentrations of GGT, can be considered to reliably reflect antemortem serum levels, thus suggesting that their postmortem values could be used as surrogates for antemortem levels. Conversely, postmortem serum (from both cardiac and femoral blood) concentrations of ALT, AST and ALP, as well as postmortem serum from right cardiac atrium blood levels of GGT, do not provide significantly accurate information on corresponding antemortem concentrations to be used as their surrogates. Generally speaking, postmortem levels of all these markers are higher in postmortem serum samples (indicating a definite though not necessarily time-dependent increase after death) and more pronouncedly in right cardiac atrium postmortem serum samples (suggesting a possible postmortem release from blood cells, for some of these markers, as well as postmortem blood movement from the liver to the right heart cavities for markers that are predominantly hepatic). 
Lastly, our results indicate that bilirubin, ALP, and ALT activity can be measured in cerebrospinal fluid samples stored in both preservative-free and sodium fluoride tubes, though ratios to blood varied widely and erratically. Globally considered, these results lead to the conclusion that cerebrospinal fluid levels of these markers are of no value to estimate their antemortem serum concentrations, irrespective of liver disease severity (and its complications) found at autopsy.

The limitations of this study must be acknowledged. The most important is the relatively small number of studied cases, which may limit the accuracy of our research. However, precise selection criteria were applied during the recruitment process in all study groups to minimize heterogeneity in the study populations. Prospective investigations including a greater number of subjects are therefore required to confirm these findings.

On the other hand, our results seem to indicate that evaluation of liver function based on postmortem laboratory investigations can be reliably performed using postmortem serum from femoral blood concentrations of bilirubin, total proteins, albumin, and GGT as surrogates for antemortem levels, which may help pathologists to produce more accurate diagnoses on liver disease and complement morphological data.

This study was not financially supported. The authors declare no conflict of interest. 
References

1) Wang FS, Fan JG, Zhang Z, Gao B, Wang HY. The global burden of liver disease: the major impact of China. Hepatology 2014;60:2099-2108.

2) Bell BP, Manos MM, Zaman A, Terrault N, Thomas A, Navarro VJ, Dhotre KB, Murphy RC, Van Ness GR, Stabach N, Robert ME, Bower WA, Bialek SR, Sofair AN. The epidemiology of newly diagnoses chronic liver disease in gastroenterology practice in the United States: results from population-based surveillance. Am J Gastroenterol 2008;103:2727-2736.

3) Knight JA. Liver function tests: their role in the diagnosis of hepatobiliary disease. J Infus Nurs $2005 ; 28: 108-117$.

4) Ramaiah SK. A toxicologist guide to the diagnostic interpretation of hepatic biochemical parameters. Food Chem Toxiol 2007;45:1551-1557.

5) Coe Jl. Postmortem chemistry update. Emphasis on forensic application. Am J Forensic Med Pathol 1993;14:91-117.

6) Coe JI. Postmortem chemistry of blood, cerebrospinal fluid, and vitreous humor. Leg Med Annu 1977;1976:55-92.

7) Uemura K, Shintani-Ishida K, Saka K, Nakajima M, Ikegaya H, Kikuchi Y, Yoshida K. Biochemical blood markers and sampling sites in forensic autopsy. J Forensic Leg Med 2008;15:312-317.

8) Schoning P, Strafuss AC. Postmortem sera and cerebrospinal fluid enzymes. J Forensic Sci 1980;25:344-348.

9) Devgun MS, Dunbar JA. Biochemical investigation of vitreous: applications in forensic medicine, especially in relation to alcohol. Forensic Sci Int 1986;31:27-34.

10) Naumann HN. Postmortem liver function tests. Am J Clin Pathol 1956;26:495-505. 
11) Michiue T, Ishikawa $T$, Kawamoto $O$, Chen JH, Wang $Q$, Zhu BL, Maeda H. Postmortem serum levels of amylase and gamma glutamyl transferase (GGT) as markers of systemic tissue damage in forensic autopsy. Leg Med (Tokyo) 2013;15:79-84.

12) Lythgoe AS. Postmortem activity of alkaline phosphatase in serum from different sites of the cadaver cardiovascular system. J Forensic Sci Soc 1981;21:337-340.

13) Arroyo A, Rosel P, Marron T. Cerebrospinal fluid: postmortem biochemical study. J Clin Forensic Med 2005;12:153-156.

14) Naumann HN, Young JM. Comparative bilirubin levels in vitreous body, synovial fluid, cerebrospinal fluid and serum after death. Proc Soc Exp Biol Med 1960;105:70-72.

15) Enticknap JB. Biochemical changes in cadaver sera. J Forensic Med 1960;7:135-146.

16) Coe Jl. Postmortem chemistries on blood: particular reference to urea nitrogen, electrolytes and bilirubin. J Forensic Sci 1974;19:33-42.

17) Hall WEB. The medico-legal application of the serum transaminase test. J Forensic Sci 1958; 3:117-122.

18) Dito WR. Transaminase activity in postmortem cerebrospinal fluid. Am J Clin Pathol 1964; 42:360-363.

19) Lythgoe AS. The activity of lactate dehydrogenase in cadaver sera: a comparison of different sampling sites. Med Sci Law 1980; 20:48-53.

20) Devgun MS, Dunbar JA. Post-mortem estimation of gamma-glutamyl transferase in vitreous humor and its association with chronic abuse of alcohol and road-traffic deaths. Forensic Sci Int 1985;28:179-180.

21) Piette M, De Schrijver G. Gamma-glutamyl transferase: applications in forensic pathology: I: Study of blood serum recovered from human bodies. Med Sci Law 1987;27:152-160. 